\title{
SINTOMAS DE DISFORIA E DEPRESSÃO EM ESTUDANTES DE ENFERMAGEM
}

Ana Patrícia Bustillos Cáceres ${ }^{1}$, Andreia Morales Cascaes², Fátima Büchele ${ }^{3}$

RESUMO: Este estudo teve como objetivo estimar a prevalência de sintomas de disforia e depressão segundo características demográficas, socioeconômicas, culturais, hábitos comportamentais e fases do curso entre os estudantes de Enfermagem. Foram pesquisados 194 estudantes do curso de Enfermagem da Universidade Federal de Santa Catarina; os dados foram coletados por meio da aplicação de um questionário e do Inventário de Depressão de Beck (IDB). A maioria era do sexo feminino (92,78\%), solteiros (91,24\%), com idade média de 22 anos. A prevalência de sintomas de disforia e depressão foi de 7,22\% e 5,67\%, respectivamente. Escolaridade da mãe, prática de lazer e religião foram associadas significativamente com sintomas de disforia e depressão $(p<0,05)$; a média dos escores obtidos do IDB foi de 8,38 (DP 7,13). O rastreamento de sinais e sintomas de disforia e depressão em estudantes torna-se importante no planejamento e monitoramento de serviços de apoio psicológico.

PALAVRAS-CHAVE: Prevalência; Depressão; Estudantes de enfermagem.

\section{SYMPTOMS OF DYSPHORIA AND DEPRESSION IN NURSING STUDENTS}

ABSTRACT: This study aimed to estimate the prevalence of symptoms of dysphoria and depression, according to demographic, socioeconomic, cultural, behavioral habits and academic phase, among Nursing students. We surveyed 194 students of the School of Nursing of Universidade Federal de Santa Catarina; data were collected through a questionnaire and the Beck Depression Inventory (BDI). Most were female (92.78\%), unmarried (91.24\%), mean age 22 years. The prevalence of symptoms of dysphoria and depression was $7.22 \%$ and $5.67 \%$ respectively. Mother's education, practice of leisure and religion were significantly associated with symptoms of dysphoria and depression $(\mathrm{p}<0.05)$, the mean score of IDB was 8.38 (SD 7.13). Screening for signs and symptoms of dysphoria and depression in students is important in planning and monitoring of psychological support services.

KEYWORDS: Prevalence; Depression; Nursing students.

\section{SÍNTOMAS DE DISFORÍA Y DEPRESIÓN EN ESTUDIANTES DE ENFERMERÍA}

RESUMEN: Este estudio tuvo el objetivo de estimar la prevalencia de síntomas de disforía y depresión según características demográficas, socioeconómicas, culturales, hábitos comportamentales y fases del curso entre los estudiantes de Enfermería. Fueron investigados 194 estudiantes del curso de Enfermería de la Universidade Federal de Santa Catarina. Los datos fueron recogidos por medio de aplicación de cuestionario y el Inventario de Depresión de Beck (IDB). La mayoría era del sexo femenino (92,78\%), solteros $(91,24 \%)$, edad media de 22 años. La prevalencia de síntomas de disforía y depresión fue 7,22\% y 5,67\%, respectivamente. Escolaridad de la madre, práctica de ocio y religión fueron asociadas de modo significativo con síntomas de disforía y depresión $(p<0,05)$; la media de los resultados obtenidos del IDB fue de 8,38 (DP 7,13). Rastrear señales y síntomas de disforía y depresión en estudiantes es importante en el planeamiento y verificación de servicios de apoyo psicológico.

PALABRAS CLAVE: Prevalencia; Depresión; Estudiantes de enfermería.

${ }^{1}$ Médica.

${ }^{2}$ Odontóloga. Mestre em Saúde Pública. Professor do Centro de Ciências da Saúde da Universidade Federal de Santa Catarina-UFSC. ${ }^{3}$ Enfermeira. Doutor em Enfermagem. Professor Adjunto do Departamento de Saúde Pública da UFSC.

\author{
Autor correspondente: \\ Fátima Büchele \\ Universidade Federal de Santa Catarina \\ Campus Universitário Trindade - 88010-970 - Florianópolis-SC-Brasil \\ E-mail: buchele@mbox1.ufsc.br
}

Recebido: 29/04/10 Aprovado: 24/08/10 


\section{INTRODUÇÃO}

Estudos demonstraram que tanto a disforia quanto a depressão são problemas que acometem, com frequência, populações de estudantes ${ }^{(1-3)}$, levando a sintomas como a diminuição de rendimento no estudo; falta de concentração, além de insônia ou sonolência constante; perda de interesse pela sua própria vida e pelo trabalho; fadiga; agitação ou retardo psicomotor; fraqueza muscular; falta de apetite e falta de energia, entre outros, o que pode levar à frustração destes estudantes e à desistência precoce do curso ${ }^{(4)}$. Além disso, a saída do seu ambiente familiar e a necessidade de enfrentamento de novos desafios muitas vezes pode ocasionar estresse aos estudantes e, por consequência, desequilíbrio emocional e estados de depressão(5).

Segundo a Organização Mundial de Saúde (OMS), a depressão é uma doença que se caracteriza por afetar o estado de humor do indivíduo, em uma combinação de tristeza, perda de interesse e diminuição de energia ${ }^{(6)}$.

No Brasil, entre 24 e 30 milhões de pessoas, em números aproximados, apresentam, apresentaram ou virão a ter, pelo menos, um episódio depressivo ao longo da vida, sendo que a prevalência é de duas a três vezes maior em mulheres do que em homens ${ }^{(7)}$. Observa-se, ainda, que a depressão pode se iniciar a partir dos cinco anos de idade; porém, em $50 \%$ dos casos, a doença tem início entre os 20 e os 50 anos, ocorrendo com maior frequência em indivíduos com problemas em seus relacionamentos afetivos ou que sejam divorciados ${ }^{(8)}$.

Já a disforia é definida como uma instabilidade persistente do humor que pode levar a períodos de depressão ou de excitação, porém, não tem gravidade suficiente para preencher os critérios diagnósticos de um transtorno depressivo ${ }^{(9)}$. Os sintomas de disforia fazem parte de um transtorno de personalidade chamado borderline ou limítrofe.

Os estudantes da área da saúde enfrentam, a cada dia, forte pressão, ocasionada por um alto nível de cobrança por parte da sociedade e do próprio curso, e pelo fato de estar em contato próximo com pacientes doentes, com prognósticos ruins e/ou deprimidos. Some-se a isso a carga horária excessiva de estudos e o trabalho que os torna vulneráveis ao desenvolvimento de transtornos como a disforia e a depressão ${ }^{(10-11)}$.

O presente estudo teve como objetivo investigar os sintomas de disforia e depressão entre os estudantes de Enfermagem da Universidade Federal de
Santa Catarina (UFSC), em Florianópolis, e descrever estes sintomas segundo características demográficas, socioeconômicas, culturais hábitos comportamentais e fases do curso.

\section{METODOLOGIA}

Tratou-se de um estudo transversal descritivo e incluiu todos os estudantes do curso de Graduação em Enfermagem da UFSC, regularmente matriculados no segundo semestre de 2008 ( $\mathrm{n}=265)$, distribuídos nos oito semestres letivos.

As variáveis independentes investigadas foram: características demográficas (sexo, idade), socioeconômicas (renda familiar, escolaridade dos pais), culturais (religião), hábitos comportamentais (lazer, prática de exercícios físicos) e período que está cursando.

O desfecho, classificado em disforia e sintomas de depressão, foi avaliado por meio do Inventário de Depressão de Beck (IDB) ${ }^{(12)}$. Este Inventário é um questionário autoavaliativo utilizado, para rastreamento de sinais e sintomas de depressão, e consiste em 21 itens, incluindo sintomas e atitudes, e cuja intensidade varia de zero a três. Os itens referem-se à tristeza, pessimismo, sensação de fracasso, falta de satisfação, sensação de culpa, sensação de punição, autodepreciação, autoacusações, ideias suicidas, crises de choro, irritabilidade, retração social, indecisão, distorção da imagem corporal, inibição para o trabalho, distúrbio do sono, fadiga, perda de apetite, perda de peso, preocupação somática e diminuição da libido ${ }^{(13-14)}$.

O desfecho foi categorizado segundo o escore obtido pela soma das pontuações de todas as questões em: (i) Sintomas ausentes: resultado menor que 15; (ii) Disforia: pontuação total entre 16 e 20; (iii) Sintomas de depressão: pontuação maior de 20. Esta forma de categorização já foi mencionada para populações não diagnosticadas por Kendall ${ }^{(15)}$, e validado por Clarice Gorenstein e Laura Andrade ${ }^{(14-16)}$, utilizando o mesmo escore para populações de universitários.

Para a coleta de dados foi aplicado um questionário autoavaliativo dividido em dois blocos. O primeiro refere-se às características demográficas (sexo, idade), socioeconômicas (renda familiar, escolaridade dos pais), culturais (religião), hábitos comportamentais (lazer, prática de exercícios físicos) e fase do curso do pesquisado; o segundo contém o IDB. Os questionários foram aplicados por uma única 
pesquisadora, no mês de setembro de 2008, ao final da aula de cada turma de Enfermagem.

A análise de dados foi conduzida no programa

Stata 9 de acordo com os seguintes procedimentos: análise descritiva exploratória do banco de dados (variável dependente e variáveis independentes), cálculos da média, mediana, desvio-padrão, amplitude para as variáveis contínuas, prevalência e intervalos de confiança de 95\% (IC95\%) para as variáveis categóricas. Para testar associações entre a variável de desfecho e as variáveis exploratórias, foi utilizado o Teste Exato de Fisher. Foram considerados estatisticamente significativos valores de $p<0,05$.

A pesquisa foi aprovada pelo Comitê de Ética em Pesquisas da Pró-Reitoria de Pesquisa e Extensão da UFSC, na data de 28 de julho de 2008 , sob número $184 / 2008$

\section{RESULTADOS}

Participaram desta pesquisa 194 estudantes, o que corresponde a uma taxa de resposta de $73,2 \%$. A maioria dos pesquisados era do sexo feminino (92,78\%), solteiros (as) (91,24\%), com idades entre 17 e 50 anos (Tabela 1), sendo que a média foi de 22 anos.

Observa-se na tabela 1 que quase a metade dos pesquisados $(45,60 \%)$ possuía renda familiar entre $\mathrm{R} \$$ 1500,00 e $\mathrm{R} \$ 5000,00$, porém, cerca de $7 \%$ recebem menos que $\mathrm{R} \$ 500,00$. O Ensino Médio prevaleceu como nível máximo de escolaridade entre os pais e mães dos entrevistados $(38,42 \%)$ e $(44,85 \%)$, respectivamente.

Tabela 1 - Frequência e porcentagem das variáveis demográficas e socioeconômicas investigadas em estudantes de Enfermagem ( $\mathrm{n}=194)$. Florianópolis, 2008

\begin{tabular}{lcc}
\hline Variáveis & \multicolumn{2}{c}{$\begin{array}{c}\text { Distribuição da amostra } \\
\text { n }\end{array}$} \\
\hline Sexo & & \\
$\quad$ Masculino & 14 & 7,22 \\
Feminino & 180 & 92,78 \\
Idade (em anos) & & \\
17 a 20 & 88 & 45,36 \\
21 a 30 & 99 & 51,03 \\
31 a 50 & 7 & 3,61 \\
Estado Civil & & \\
Solteiro (a) & 177 & 91,24 \\
Casado (a) & 16 & 8,25 \\
Separado (a) & 1 & 0,52 \\
Viúvo (a) & - & - \\
\hline
\end{tabular}

\section{Renda Familiar (em Reais)}

$\begin{array}{lcc}0|---| 500,00 & 13 & 6,74 \\ 500,00--\mid 1.500,00 & 60 & 31,09 \\ 1.500,00--|| 5.000,00 & 88 & 45,60 \\ 5.000,00 \text { ou mais } & 32 & 16,58\end{array}$

\section{Nível de Escolaridade do Pai}

$\begin{array}{lcc}\text { Analfabeto } & 2 & 1,05 \\ \text { Ensino Fundamental } & 40 & 21,05 \\ \text { Ensino Médio } & 73 & 38,42 \\ \text { Ensino Superior } & 48 & 25,26 \\ \text { Pós-Graduação } & 27 & 14,21 \\ \text { Nível de Escolaridade da Mãe } & & \\ \text { Analfabeta } & 1 & 0,52 \\ \text { Ensino Fundamental } & 36 & 18,56 \\ \text { Ensino Médio } & 87 & 44,85 \\ \text { Ensino Superior } & 45 & 23,20 \\ \text { Pós-Graduação } & 25 & 12,89\end{array}$

Em relação à religião, uma parcela grande (57,22\%) é católica. Chama atenção que $25 \%$ dos pesquisados não realiza atividades de lazer regularmente, assim como a prática de atividades físicas não é exercida por quase $60 \%$ dos estudantes. Dentre aqueles que praticam atividades físicas, quase a metade as realiza regularmente de duas a três vezes na semana (Tabela 2). Outras atividades de lazer informadas pelos pesquisados foram: ir ao cinema, sair com o(a) namorado(a), sair com amigos, visitar a família, ir à praia e tocar violão.

Tabela 2 - Frequência e porcentagem das variáveis culturais e comportamentais investigadas em estudantes de Enfermagem ( $\mathrm{n}=194)$. Florianópolis, 2008

\begin{tabular}{lcc}
\hline Variáveis & $\begin{array}{c}\text { Distribuição da amostra } \\
\text { n }\end{array}$ \\
\hline Religião & & \\
$\quad$ Católico & 111 & 57,22 \\
$\quad$ Evangélico & 21 & 10,82 \\
$\quad$ Espírita & 45 & 23,20 \\
$\quad$ Outras & 17 & 8,76 \\
$\quad$ Realização de atividades & & \\
de lazer & & \\
$\quad$ Sim & 143 & 73,71 \\
$\quad$ Não & 51 & 26,29 \\
Prática de atividades físicas & & \\
$\quad$ Sim & 81 & 41,75 \\
$\quad$ Não & 113 & 58,25 \\
\hline & & Continua
\end{tabular}


Continuação

\begin{tabular}{lcc}
\hline $\begin{array}{l}\text { Frequência de atividades } \\
\text { físicas }\end{array}$ & & \\
\hline Um vez no mês & 4 & 4,94 \\
A cada 15 dias & 4 & 4,94 \\
Semanalmente & 15 & 18,52 \\
2 a 3 vezes na semana & 40 & 49,38 \\
Diariamente & 18 & 22,22 \\
Período do curso & & \\
$1^{\circ}$ ano & 54 & 28,13 \\
$2^{\circ}$ ano & 57 & 29,69 \\
$3^{\circ}$ ano & 34 & 17,71 \\
$4^{\circ}$ ano & 47 & 24,48 \\
\hline
\end{tabular}

A prevalência dos sintomas de disforia entre os estudantes de Enfermagem foi de 7,22\%, enquanto que a dos sintomas de depressão foi de 5,67\%. A média dos escores obtidos por meio do IDB foi de 8,38 (DP 7,13) e a mediana 7,0 (Tabela 3).

A escolaridade da mãe, religião e realização de atividade de lazer apresentaram associação estatisticamente significativa com os sintomas de disforia e depressão $(\mathrm{p}<0,05)$ (Tabela 4).

Tabela 3 - Associação entre sintomas de disforia e de depressão e variáveis demográficas e socioeconômicas investigadas em estudantes de Enfermagem (n=194). Florianópolis, 2008

\begin{tabular}{|c|c|c|c|c|c|c|c|}
\hline \multirow[t]{2}{*}{ Variáveis } & \multicolumn{2}{|c|}{ Sintomas ausentes } & \multicolumn{2}{|c|}{$\begin{array}{l}\text { Sintomas de } \\
\text { disforia }\end{array}$} & \multicolumn{2}{|c|}{$\begin{array}{l}\text { Sintomas de } \\
\text { depressão }\end{array}$} & \multirow[t]{2}{*}{ Valor-p* } \\
\hline & $\mathbf{n}$ & $\%$ & $\mathbf{n}$ & $\%$ & $\mathbf{n}$ & $\%$ & \\
\hline & 169 & 87,11 & 14 & 7,22 & 11 & 5,67 & \\
\hline Sexo & & & & & & & $\mathbf{0 , 8 3 1}$ \\
\hline Masculino & 12 & 85,71 & 1 & 7,14 & 1 & 7,14 & \\
\hline Feminino & 157 & 87,22 & 13 & 7,22 & 10 & 5,56 & \\
\hline Idade (em anos) & & & & & & & 0,659 \\
\hline 17 a 20 & 79 & 89,77 & 5 & 5,68 & 4 & 4,55 & \\
\hline 21 a 30 & 84 & 84,85 & 8 & 8,08 & 7 & 7,07 & \\
\hline 31 a 50 & 6 & 85,71 & 1 & 14,29 & - & - & \\
\hline Estado Civil & & & & & & & 1,000 \\
\hline Solteiro (a) & 154 & 87,01 & 13 & 7,34 & 10 & 5,65 & \\
\hline Casado (a) & 14 & 87,50 & 1 & 6,25 & 1 & 6,25 & \\
\hline Separado (a) & 1 & 100,00 & - & - & - & - & \\
\hline Viúvo (a) & - & - & - & - & - & - & \\
\hline Renda Familiar (em Reais) & & & & & & & 0,352 \\
\hline $0---500,00$ & 10 & 76,92 & 3 & 23,08 & 0 & 0,00 & \\
\hline $500,00---\mid 1.500,00$ & 51 & 85,00 & 4 & 6,67 & 5 & 8,33 & \\
\hline $1.500,00---\mid 5.000,00$ & 79 & 89,77 & 5 & 5,68 & 4 & 4,55 & \\
\hline $5.000,00$ ou mais & 29 & 90,63 & 1 & 3,13 & 2 & 6,25 & \\
\hline Escolaridade do Pai & & & & & & & 0,173 \\
\hline Analfabeto & 1 & 50,00 & - & - & 1 & 50,00 & \\
\hline Ensino Fundamental & 37 & 92,50 & 2 & 5,00 & 1 & 2,50 & \\
\hline Ensino Médio & 60 & 82,19 & 8 & 10,96 & 5 & 6,85 & \\
\hline Ensino Superior & 43 & 89,58 & 4 & 8,33 & 1 & 2,08 & \\
\hline Pós-Graduação & 25 & 92,59 & - & - & 2 & 7,41 & \\
\hline Escolaridade da Mãe & & & & & & & 0,04 \\
\hline Analfabeta & 1 & 100,00 & - & - & - & - & \\
\hline Ensino Fundamental & 30 & 83,33 & 4 & 11,11 & 2 & 5,56 & \\
\hline Ensino Médio & 79 & 90,80 & 2 & 2,30 & 6 & 6,90 & \\
\hline Ensino Superior & 38 & 84,44 & 7 & 15,56 & - & - & \\
\hline Pós-Graduação & 21 & 84,00 & 1 & 4,00 & 3 & 12,00 & \\
\hline
\end{tabular}

*Teste Exato de Fisher 
Tabela 4 - Associação entre sintomas de disforia e de depressão e variáveis culturais e comportamentais investigadas em estudantes de Enfermagem ( $\mathrm{n}=194)$. Florianópolis, 2008

\begin{tabular}{|c|c|c|c|c|c|c|c|}
\hline \multirow[t]{2}{*}{ Variáveis } & \multicolumn{2}{|c|}{ Sintomas ausentes } & \multicolumn{2}{|c|}{$\begin{array}{l}\text { Sintomas de } \\
\text { disforia }\end{array}$} & \multicolumn{2}{|c|}{$\begin{array}{c}\text { Sintomas de } \\
\text { depressão }\end{array}$} & \multirow[t]{2}{*}{$\underset{*}{\text { Valor-p }}$} \\
\hline & $\mathbf{n}$ & $\%$ & n & $\%$ & $\mathbf{n}$ & $\%$ & \\
\hline Religião & & & & & & & 0,047 \\
\hline Católico & 101 & 90,99 & 7 & 6,31 & 3 & 2,70 & \\
\hline Evangélico & 18 & 85,71 & 1 & 4,76 & 2 & 9,52 & \\
\hline Espírita & 38 & 84,44 & 5 & 11,11 & 2 & 4,44 & \\
\hline Outras & 12 & 70,59 & 1 & 5,88 & 4 & 23,53 & \\
\hline Realização de atividades de lazer & & & & & & & 0,004 \\
\hline Sim & 131 & 91,61 & 8 & 5,59 & 4 & 2,80 & \\
\hline Não & 38 & 74,51 & 6 & 11,76 & 7 & 13,73 & \\
\hline Prática de atividades físicas & & & & & & & 0,931 \\
\hline Sim & 71 & 87,65 & 6 & 7,41 & 4 & 4,94 & \\
\hline Não & 98 & 86,73 & 8 & 7,08 & 7 & 6,19 & \\
\hline Frequência de atividades físicas & & & & & & & $\mathbf{0 , 8 3 7}$ \\
\hline Um vez no mês & 4 & 100,00 & - & - & - & - & \\
\hline A cada 15 dias & 4 & 100,00 & - & - & - & - & \\
\hline Semanalmente & 13 & 86,67 & 2 & 13,33 & - & - & \\
\hline 2 a 3 vezes na semana & 33 & 82,50 & 3 & 7,50 & 4 & 10,00 & \\
\hline Diariamente & 17 & 94,44 & 1 & 5,56 & - & - & \\
\hline Período do curso & & & & & & & 0,993 \\
\hline $1^{\circ}$ ano & 47 & 87,04 & 4 & 7,40 & 3 & 5,56 & \\
\hline $2^{\circ}$ ano & 51 & 89,48 & 3 & 5,26 & 3 & 5,26 & \\
\hline $3^{\circ}$ ano & 29 & 85,29 & 3 & 8,83 & 2 & 5,88 & \\
\hline $4^{\circ}$ ano & 40 & 85,11 & 4 & 8,51 & 3 & 6,38 & \\
\hline
\end{tabular}

*Teste Exato de Fisher

\section{DISCUSSÃO}

A depressão é um problema de saúde pública, sendo considerada uma das principais causas de incapacitação no mundo, que pode ocasionar limitação do funcionamento social, físico e pessoal. Estudo realizado em $1998^{(17)}$ mostrou que pessoas da equipe de um hospital geral não estavam preparadas para atender pacientes com manifestações de distúrbio emocional. Estudos como esse revelam a necessidade de discutir temas relacionados a transtornos psiquiátricos.

A presente pesquisa fez uso do Inventário de Depressão de Beck, largamente utilizado na clínica em pesquisas em diversos locais do mundo ${ }^{(4-6,11)} \mathrm{e}$ validado no Brasil por Gorenstein \& Andrade ${ }^{(14-16)}$. O $\mathrm{IDB}^{(12)}$ foi desenvolvido com o objetivo de mensurar as manifestações comportamentais da depressão, bem como a intensidade dos sintomas depressivos, sendo instrumento adequado para o uso em pacientes psiquiátricos. Porém, tem sido utilizado em pesquisas, tanto com pacientes não psiquiátricos como na população em geral ${ }^{(13)}$. Este inventário não tem pretensão diagnóstica, e sim de rastreamento; é um instrumento fidedigno de rápida aplicação, válido e autoadministrado $^{(10)}$

Nos estudantes de Enfermagem investigados no presente estudo, obteve-se uma prevalência de sintomas de disforia de 7,22\% e de depressão de 5,67\%. Estudo realizadoem várias universidades da Europa demonstrou prevalência de sintomas depressivos em mulheres e homens, respectivamente, conforme a seguir: na Alemanha 26,7\% e 22,8\%; na Dinamarca, 24,9\% e 12,1\%; na Polônia, 45,5\% e 27,3\%; e, na Bulgária, de $42,9 \%$ e $33,8 \%{ }^{(5)}$. Cabe ressaltar que neste estudo foi utilizado o IDB modificado, com 20 perguntas do inventário original, tendo sido removida a pergunta sobre "perda de peso", por não ter sido considerada específica, e ponto de corte acima de 35 pontos.

No Brasil, algumas pesquisas também têm relatado os sintomas depressivos em estudantes uni- 
versitários. Um estudo realizado na cidade de Tubarão, Santa Catarina no ano de 2007 com estudantes de Medicina, demonstrou prevalência de sintomas depressivos em $27 \%$ dos pesquisados; foi utilizado um ponto de corte empregado para populações previamente diagnosticadas com depressão, as quais determinaram sintomas depressivos com escore maior do que dez ${ }^{(18)}$.

Pesquisa com estudantes da Escola de Enfermagem da Universidade de São Paulo-Ribeirão Preto demonstrou uma prevalência de sintomas de disforia de $10,3 \%$, e de depressão de $6,7 \%$; semelhante às do presente estudo. Além disso, utilizou um ponto de corte próximo ao do presente estudo, considerando sintomas de disforia a soma dos escores do IBD menor de 15 a 20, e sintomas de depressão soma maior que $20^{(4)}$.

Na cidade de São Paulo, outro estudo realizado com estudantes de Enfermagem, utilizando o mesmo ponto de corte para o escore do IDB, avaliou os sintomas de depressão, demonstrando uma prevalência de $26,06 \%$, o que se aproxima dos valores obtidos em estudos em países europeus ${ }^{(5,9,19)}$. Uma possível explicação deste resultado poderia ser a ausência de um serviço de ajuda psicológica aos estudantes. Isto não ocorre na Universidade Federal de Santa Catarina, que, além de possuir um programa de apoio psicológico aos estudantes de Enfermagem, possui também na grade curricular disciplinas que contribuem neste sentido, como é caso da disciplina de Aprendizagem Vivencial.

É conhecido que atividades de lazer e físicas são muito importantes para evitar sintomas de disforia e depressão uma vez que, teoricamente, a prática destas atividades estimula a produção e liberação de serotonina, ocasionando sensação de bem-estar físico e psicológico ${ }^{(20)}$. É possível observar que, no presente estudo, a prevalência de sintomas de disforia foi mais que o dobro e os de depressão foi cerca de cinco vezes maior entre estudantes que não realizavam atividades de lazer. Já os sintomas de disforia e depressão, em relação à prática de atividade física, foram semelhantes entre os dois grupos, porém, sem associações significativas. Entretanto, em estudos transversais, como o presente, em virtude da possibilidade de causalidade reversa, não se pode afirmar a direção de causalidade nos achados, isto é, se a prática da atividade física ocorre porque os indivíduos estão mais deprimidos, ou se não ocorre em virtude destes sintomas.

A prevalência dos sintomas de depressão foi maior entre os casados; apesar de não apresentar valor estatisticamente significativo, sugere-se que maior prevalência entre os estudantes casados poderia ocorrer em decorrência da responsabilidade que estes assumem em casa.

Os estudantes com idade entre 21 e 50 anos apresentaram prevalência de depressão de 7,07\%. Estudo realizado na Turquia demonstrou que estudantes com idade superior a 22 anos apresentavam maior prevalência de sintomas depressivos ${ }^{(15)}$. O mesmo estudo demonstrou prevalência de $34,7 \%$ de sintomas depressivos entre estudantes com baixa renda e de $21,9 \%$ entre aqueles com alta renda; o mesmo pode ser observado no presente estudo. Estudantes com menor renda podem apresentar maiores dificuldades para se manter e estudar, bem como ter acesso a alimentação saudável e prática de atividades físicas e de lazer, quando comparados a estudantes com maior renda.

Em relação aos sintomas depressivos, observamos um aumento a partir do segundo ano de curso. Pesquisadores verificaram prevalência de sintomas depressivos maior no último ano $(42,10 \%)$ quando comparado com o primeiro (17,9\%), sugerindo que, com o aumento da carga horária e exigências do curso, os estudantes tendem a apresentar maior prevalência de sintomas de disforia e depressão $0^{(19)}$.

Podemos observar que $12,89 \%$ dos pesquisados apresentaram intenções suicidas alguma vez. A importância que damos a isto é pelo fato de que a taxa de suicídios, em geral, pode ser considerada como um indicador da prevalência da depressão grave ${ }^{(12)}$.

Em relação às limitações do estudo, podemos considerar que a principal delas refere-se ao delineamento transversal, que não permitiu observar relação de causalidade entre as variáveis investigadas, possibilitando a ocorrência de viés de causalidade reversa. O viés de seleção pode ter ocorrido em decorrência da porcentagem de perdas no estudo, o que poderia subestimar as prevalências encontradas. Pelo fato da depressão ser considerada um problema de saúde pública, torna-se importante rastrear os sinais e sintomas de disforia e depressão nas populações, propondo a formulação de soluções de acordo com as necessidades.

\section{CONSIDERAÇÕES FINAIS}

Os estudantes da área de saúde convivem precocemente com a dor humana durante a sua formação, o que pode trazer uma maior probabilidade de desenvolver transtornos do humor, como a disforia 
e a depressão. O serviço de apoio psicológico que esses estudantes têm na Universidade Federal de Santa Catarina, além das disciplinas que enfocam a importância da saúde mental, pode ter contribuído para a prevalência encontrada.

Esse estudo aponta para a necessidade de programas que acompanhem, efetivamente, o aluno durante a graduação. Dessa forma, sugere-se o monitoramento dos sintomas de disforia e depressão ao longo do curso, com intuito de ampliar e melhorar o serviço de apoio psicológico ao estudante e, consequentemente, sua qualidade de vida.

Também fica evidente que estudantes e professores precisam estar atentos para sinais de disforia e depressão. Estratégias educacionais podem contribuir para o diagnóstico precoce e orientações frente a esses problemas.

\section{AGRADECIMENTOS}

Agradecemos aos estudantes que participaram do estudo e ao Departamento de Enfermagem da UFSC pela colaboração.

\section{REFERÊNCIAS}

1. Steptoe A, Tsuda A, Tanaka Y, Wardle J. Depressive symptoms, socio-economic background, sense of control and cultural factors in university students from 23 countries. Inter J Behav Med. 2007;14(2):97-107.

2. Furegato ARP, Santos JLF, Silva EC. Depressão entre estudantes de enfermagem relacionada à auto-estima, à percepção da sua saúde e interesse por saúde mental. Rev Latino-Am Enfermagem. 2008;16(2):1-7.

3. Garro IMB, Camillo SO, Nóbrega MPSS. Depressão em graduandos de enfermagem. Acta Paul Enferm. 2006;19(2):162-7.

4. Furegato ARF, Silva EC, Campos MC, Cassiano RP. Depressão e auto-estima entre acadêmicos de enfermagem. Rev Psiquiatr Clin. 2006;33(5):239-44.

5. Figueiredo RM, Oliveira MAP. Necessidades de estudantes universitários para implantação de um serviço de orientação e educação em saúde mental. Rev Latino-Am Enfermagem. 1995;3(1):5-18.

6. World Health Organization (WHO). Conquering depression: you can get out of the blues. Regional Office for South-East Asia: World Health Organization; 2001.
7. Souza JC, Guimarães LAM, Ballone GJ. Psicopatologia e psiquiatria básicas. São Paulo: Vetor; 2004.

8. Sadock BJ. Transtornos do humor. In: Sadock BJ, Sadock VA. Compêndio de psiquiatria: ciências do comportamento e psiquiatria clínica. $9^{\mathrm{a}} \mathrm{ed}$. Porto Alegre: Artmed; 2007. p.572-629.

9. Rey L. Dicionário de termos técnicos de medicina e saúde. $2^{\mathrm{a}}$ ed. Rio de Janeiro: Guanabara Koogan, 2003.

10. Cunha JA, Freitas NK, Raymundo MGB. Catálogo de técnicas úteis. In: Cunha JA. Psicodiagnóstico-R. $4^{\mathrm{a}}$ ed. Porto Alegre: Artes Médicas; 1993. p. 135-6.

11. Moro A, Valle JB, Lima LP. Sintomas depressivos nos estudantes de medicina da região de Joinville (SC). Rev Bras Educ Med. 2005;29(2):97-102.

12. Beck AT, Rush AJ, Shaw BF, Emery G. Terapia cognitiva da depressão. Porto Alegre: Artes Médicas; 1997.

13. Beck AT, Ward CH, Mendelson M, Mock J, Erbaugh G. An inventory for measuring depression. Arch Gen Psych. 1961;4(6):53-63.

14. Gorenstein C, Andrade L. Inventário de Depressão de Beck: propriedades psicométricas da versão em português. Rev Psiquiatr Clin. 1998;25(5):245-50.

15. Kendall PC, Hollon SD, Beck AT, Hammen CL, Ingram RE. Issues and recommendations regarding use of the Beck Depression Inventory. Cogn Ther Res.1987;11(3):289-99.

16. Gorenstein C, Andrade L. Version of a portuguese version of the Beck Depression Inventory and the State-Trait Anxiety Inventory in brazilian subjects. Braz J Med Bio Res. 1996;29(4):453-57.

17. Stefanelli MC, Bardelli MH, Silva IA. Percepções e reações do pessoal da equipe de enfermagem, de hospital geral, às manifestações de distúrbio emocional de pacientes internados. Cogitare Enferm 1998;3(1):97104.

18. Nunes MF, Junqueira A. Sintomas depressivos nos estudantes de medicina da Universidade do Sul de Santa Catarina - UNISUL [trabalho de conclusão de curso] Tubarão (SC): Universidade do Sul de Santa Catarina; 2007.

19. Bostanci M, Ozdel O, Oguzhanoglu NK, Ozdel L, Ergin A, Ergin N, et al. Depressive symptomatology among university students in Denizli, Turkey: 
prevalence and sociodemographic correlates. Cro Med J. 2005;46(1):96-100.

20. Braunwald E, Faucy AS, Kasper DL, Hauser SL, Longo DL, Jameson JL. Harrison medicina interna. $15^{\mathrm{a}}$. ed. Rio de Janeiro: McGraw-Hill Interamericana do Brasil: 2002. 